\title{
MIXED FINITE ELEMENT FORMULATIONS FOR POLYCONVEX ANISOTROPIC MATERIAL FORMULATIONS
}

\author{
Julian Dietzsch $^{1}$ and Michael Groß ${ }^{2}$ \\ ${ }^{1}$ Technische Universität Chemnitz, Professorship of applied mechanics and dynamics, Reichenhainer \\ Straße 70, D-09126 Chemnitz, julian.dietzsch@mb.tu-chemnitz.de \\ 2 michael.gross@mb.tu-chemnitz.de
}

Key words: Finite element method, mixed finite elements, energy-momentum conserving, locking behaviour, nearly incompressible elasticity, anisotropy.

\begin{abstract}
Our research activity takes place within the research project GR 3297/4, funded by 'Deutsche Forschungsgemeinschaft' (DFG), and aims at a robust simulation method for fiber-reinforced materials in light-weight structures. One goal is to avoid looking-effects in the static and dynamic regime, which occur due to nearly incompressible matrix materials and highly stiff fibers. Therefore, we extend the mixed finite elment formulations, hown in References $[1,2,3]$. In the description of the material behavior, we also use polyconvex strain energy functions [4]. In case of the so-called CoFEM element in Reference [2], the volumetric dilatation and the cofactor of the right Cauchy-Green tensor are approximated independently beside the displacement. In Reference [3], this formulation is extended so, that the right Cauchy-Green tensor of the anisotropic strain energy function is also approximated independently. In this presentation, we also approximate the cofactor and the volumetric dilatation of the anisotropic right Cauchy-Green tensor independently. We analyse the spatial convergence of the new mixed finite elements for hexahedral elements up to a cubic approximation in space. Thereby, we look especially at the different possible combinations of polynomial degrees of the independent mixed variables and the impact of this on the efficiency of the simulation (see Reference [5]). As numerical examples serve the well-known cooks cantilever beam and an axisymmetric pipe. Hereby, the bodies have different materials domains with different material parameters and fiber directions.
\end{abstract}

\section{CONTINUUM MODEL}

As continuum model, we consider an anisotropic material with $n_{\mathrm{F}}$ fiber directions $\boldsymbol{a}_{0}^{i}$ moving in the Euclidean space $\mathbb{R}^{n_{\mathrm{dim}}}$ with the constant ambient temperature $\Theta_{\infty}$. The strain energy function of the material with a thermo-viscoelastic matrix and thermoelastic fibers is given by

$$
\Psi\left(\boldsymbol{C}, \boldsymbol{C}_{v}, \Theta\right)=\Psi_{\mathrm{M}}\left(\boldsymbol{C}, \boldsymbol{C}_{v}, \Theta\right)+\sum_{i=1}^{n_{\mathrm{F}}} \Psi_{\mathrm{F}_{i}}\left(\boldsymbol{C}, \Theta, \boldsymbol{M}_{i}\right),
$$

which is split into a single matrix part $\Psi_{\mathrm{M}}$ and multiple $n_{\mathrm{F}}$ fiber parts $\Psi_{\mathrm{F}_{i}}$. Here, $\boldsymbol{M}_{i}=\boldsymbol{a}_{0}^{i} \otimes \boldsymbol{a}_{0}^{i}$ define the structural tensor, $\boldsymbol{C}=\boldsymbol{F}^{T} \boldsymbol{F}$ define the right Cauchy-Green tensor, $\boldsymbol{C}_{v}$ define the viscous right CauchyGreen tensor and $\Theta$ define the absolute temperature. With the volume dilatation $J(\boldsymbol{C})=\operatorname{det}[\boldsymbol{F}]=\sqrt{\operatorname{det}[\boldsymbol{C}]}$, 
we assume the specific dependencies:

$$
\begin{aligned}
& \Psi_{\mathrm{M}}\left(\boldsymbol{C}, \operatorname{cof}[\boldsymbol{C}], J, \Theta, \boldsymbol{C}_{v}\right)=\Psi_{\mathrm{M}}^{\mathrm{iso}}(\boldsymbol{C}, \operatorname{cof}[\boldsymbol{C}], J)+\Psi_{\mathrm{M}}^{\mathrm{vol}}(J)+\Psi_{\mathrm{M}}^{\mathrm{cap}}(\Theta)+\Psi_{\mathrm{M}}^{\mathrm{coup}}(\Theta, J)+\Psi_{\mathrm{M}}^{\mathrm{vis}}(\boldsymbol{\Lambda}) \\
& \Psi_{\mathrm{F}_{i}}\left(\boldsymbol{C}, \operatorname{cof}[\boldsymbol{C}], J, \Theta, \boldsymbol{M}_{i}\right)=\Psi_{\mathrm{F}_{i}}^{\text {ela }}\left(\boldsymbol{C}, \operatorname{cof}[\boldsymbol{C}], J, \boldsymbol{M}_{i}\right)+\Psi_{\mathrm{F}_{i}}^{\text {cap }}(\Theta)+\Psi_{\mathrm{F}_{i}}^{\text {coup }}\left(\Theta, \boldsymbol{C}, \boldsymbol{M}_{i}\right)
\end{aligned}
$$

The elastic part of the matrix function $\Psi_{\mathrm{M}}$ is split into an isochoric part $\Psi_{\mathrm{M}}^{\text {iso }}$ and a volumetric part $\Psi_{\mathrm{M}}^{\mathrm{vol}}$. We subdivided the thermo-elastic free energy of the matrix into a heat capacity part $\Psi_{\mathrm{M}}^{\text {cap }}$ and the part of the thermo-mechanical coupling effect $\Psi_{\mathrm{M}}^{\text {coup }}$, where $\beta_{\mathrm{M}}$ ist the coefficient of linear thermal expansion for the matrix. The thermal part of the fiber free energy is separated in the same manner. We consider a heat capacity function $\Psi_{\mathrm{F}_{i}}^{\text {cap }}$ and the function of the thermo-mechanical coupling $\Psi_{\mathrm{F}_{i}}^{\text {coup }}$ with the coefficients of linear thermal expansion $\beta_{\mathrm{F}_{i}}$ and the fourth invariant $I_{4}^{i}=\operatorname{tr}\left[\boldsymbol{C M}_{i}\right]$. Both coupling parts are given by

$$
\Psi_{\mathrm{M}}^{\text {coup }}=-2 n_{\mathrm{dim}} \beta_{\mathrm{M}}\left(\Theta-\Theta_{\infty}\right) J \frac{\partial \Psi_{\mathrm{M}}^{\mathrm{vol}}(J)}{\partial J} \quad \Psi_{\mathrm{F}_{i}}^{\text {coup }}=-2 \beta_{\mathrm{F}_{i}}\left(\Theta-\Theta_{\infty}\right) \sqrt{I_{4}^{i}} \frac{\partial \Psi_{\mathrm{F}_{i}}^{\text {ela }}\left(I_{4}^{i}, \ldots\right)}{\partial I_{4}^{i}}
$$

The function $\Psi_{\mathrm{M}}^{\mathrm{vis}}$ is the viscoelastic free energy function of the matrix material, with $\Lambda=C C_{v}^{-1}$.

\section{FINITE ELEMENT FORMULATION}

The finite element discretization follows from the mixed principle of virtual power (see Reference [8]. Here, we need the complete internal energy, which consists of the assumed temperature field $\tilde{\Theta}$, the entropy density field $\eta$ as the corresponding Lagrange multiplier, the superimposed stress tensor $\tilde{S}$ to multiplier S. The internal
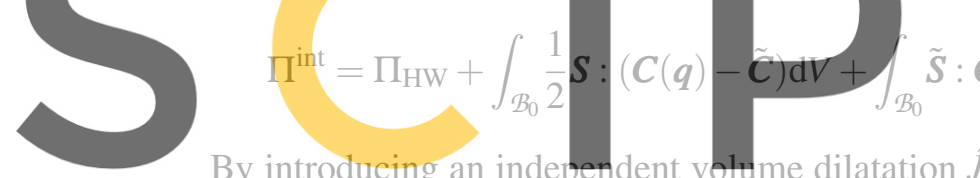

By introducing an inderendent volume dilatation $J$
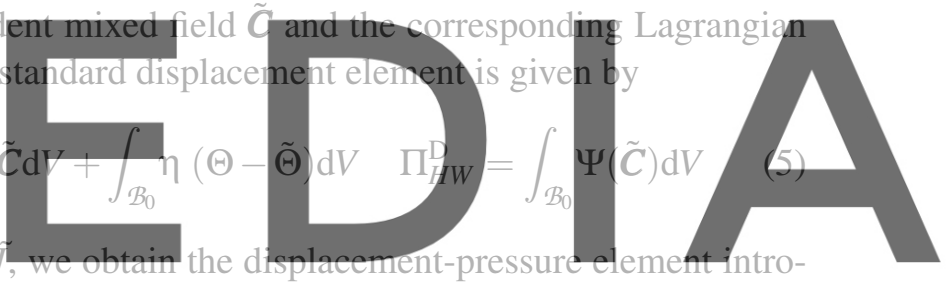

duced by Simo et al. [1]. Here, the Lagrange multiplier $p$ plays the role of the hydrostatic pressure and

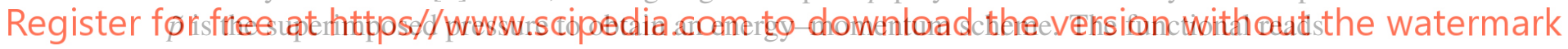

$$
\Pi_{\mathrm{HW}}^{\mathrm{DP}}=\Pi_{\mathrm{HW}}^{\mathrm{D}}+\int_{\mathcal{B}_{0}} p(J(\tilde{\boldsymbol{C}})-\tilde{J}) \mathrm{d} V+\int_{\mathcal{B}_{0}} \tilde{p} \tilde{J} \mathrm{~d} V
$$

with $\Psi_{\mathrm{M}}\left(\tilde{\boldsymbol{C}}, \operatorname{cof}[\tilde{\boldsymbol{C}}], \tilde{J}, \Theta, \boldsymbol{C}_{v}\right)$ and $\Psi_{\mathrm{F}_{i}}\left(\tilde{\boldsymbol{C}}, \operatorname{cof}[\tilde{\boldsymbol{C}}], \tilde{J}, \Theta, \boldsymbol{M}_{i}\right)$. A third functional is shown in Reference [2]. Here an additional field for the cofactor of $\boldsymbol{C}$ and the corresponding superimposed stress tensor $\tilde{\boldsymbol{B}}$ is introduced, such that we arrive at the functional

$$
\Pi_{\mathrm{HW}}^{\mathrm{CoFEM}}=\Pi_{\mathrm{HW}}^{\mathrm{DP}}+\int_{\mathcal{B}_{0}} \boldsymbol{B}:(\operatorname{cof}[\tilde{\boldsymbol{C}}]-\tilde{\boldsymbol{H}}) \mathrm{d} V+\int_{\mathcal{B}_{0}} \tilde{\boldsymbol{B}}: \tilde{\boldsymbol{H}} \mathrm{d} V
$$

with $\Psi_{\mathrm{M}}\left(\tilde{\boldsymbol{C}}, \tilde{\boldsymbol{H}}, \tilde{J}, \Theta, \boldsymbol{C}_{v}\right)$ and $\Psi_{\mathrm{F}_{i}}\left(\tilde{\boldsymbol{C}}, \tilde{\boldsymbol{H}}, \tilde{J}, \Theta, \boldsymbol{M}_{i}\right)$. Especially for anisotropic material formulations the SKA element is presented in Reference [3], which introduces the field $\tilde{\boldsymbol{C}}_{A}$ for the anisotropic part $\Psi_{\mathrm{F}_{i}}$. The anisotropic part of the stress tensor is represented by the Lagrange multiplier $\boldsymbol{S}_{A}$ and the superimposed stress tensor by $\tilde{\boldsymbol{S}}_{A}$. Here, we arrive at

$$
\Pi_{\mathrm{HW}}^{\mathrm{CoSKA}}=\Pi_{\mathrm{HW}}^{\mathrm{CoFEM}}+\int_{\mathcal{B}_{0}} \frac{1}{2} \boldsymbol{S}_{A}:\left(\tilde{\boldsymbol{C}}-\tilde{\boldsymbol{C}}_{A}\right) \mathrm{d} V+\int_{\mathcal{B}_{0}} \tilde{\boldsymbol{S}}_{A}: \tilde{\boldsymbol{C}}_{A} \mathrm{~d} V
$$


Table 1: Dependencies of the different parts of the strain energy function for the CoCoA element formulation.

\begin{tabular}{l|l|l|l}
\hline$\tilde{\boldsymbol{S}}$ & $\tilde{\Psi}=\Psi_{\mathrm{M}}^{\text {iso }}(\tilde{\boldsymbol{C}})+\Psi_{\mathrm{M}}^{\mathrm{cap}}(\Theta)+\Psi_{\mathrm{M}}^{\mathrm{vis}}(\boldsymbol{\Lambda})+\sum_{i=1}^{n_{\mathrm{F}}}\left[\Psi_{\mathrm{F}_{i}}^{\text {cap }}(\Theta)\right]$ & $\tilde{\boldsymbol{B}}$ & $\tilde{\Psi}=\Psi_{\mathrm{M}}^{\text {iso }}(\tilde{\boldsymbol{H}})$ \\
\hline$\tilde{p}$ & $\tilde{\Psi}=\Psi_{\mathrm{M}}^{\text {iso }}(\tilde{\boldsymbol{J}})+\Psi_{\mathrm{M}}^{\mathrm{vol}}(\tilde{\boldsymbol{J}})+\Psi_{\mathrm{M}}^{\mathrm{coup}}(\Theta, \tilde{J})$ & $\tilde{\boldsymbol{B}}_{A}$ & $\tilde{\Psi}=\sum_{i=1}^{n_{\mathrm{F}}}\left[\Psi_{\mathrm{F}_{i}}^{\text {ela }}\left(\tilde{\boldsymbol{H}}_{A}, \boldsymbol{M}_{i}\right)\right]$ \\
\hline$\tilde{\boldsymbol{S}}_{A}$ & $\tilde{\Psi}=\sum_{i=1}^{n_{\mathrm{F}}}\left[\Psi_{\mathrm{F}_{i}}^{\text {ela }}\left(\tilde{\boldsymbol{C}}_{A}, \boldsymbol{M}_{i}\right)+\Psi_{\mathrm{F}_{i}}^{\text {coup }}\left(\Theta, \tilde{\boldsymbol{C}}_{A}, \boldsymbol{M}_{i}\right)\right]$ & $\tilde{p}_{A}$ & $\tilde{\Psi}=\sum_{i=1}^{n_{\mathrm{F}}}\left[\Psi_{\mathrm{F}_{i}}^{\text {ela }}\left(\tilde{J}_{A}, \boldsymbol{M}_{i}\right)\right]$ \\
\hline
\end{tabular}

with $\Psi_{\mathrm{M}}\left(\tilde{\boldsymbol{C}}, \tilde{\boldsymbol{H}}, \tilde{J}, \Theta, \boldsymbol{C}_{v}\right)$ and $\Psi_{\mathrm{F}_{i}}\left(\tilde{\boldsymbol{C}}_{A}, \operatorname{cof}\left[\tilde{\boldsymbol{C}}_{A}\right], \sqrt{\operatorname{det} \tilde{\boldsymbol{C}}_{A}}, \Theta, \boldsymbol{M}_{i}\right)$. Finally, we add the fields $\tilde{\boldsymbol{H}}_{A}$ and $\tilde{J}_{A}$, the corresponding Lagrange multipliers $\boldsymbol{B}_{A}$ and $p_{A}$ and the superimposed fields $\tilde{\boldsymbol{B}}_{A}$ and $\tilde{p}_{A}$. Thus, we obtain the CoCoA element, as shown in Reference [5], by the means of the functional

$$
\Pi_{\mathrm{HW}}^{\mathrm{CoCoA}}=\Pi_{\mathrm{HW}}^{\mathrm{CoSKA}}+\int_{\mathcal{B}_{0}} \boldsymbol{B}_{A}:\left(\operatorname{cof}[\tilde{\boldsymbol{C}}]-\tilde{\boldsymbol{H}}_{A}\right) \mathrm{d} V+\int_{\mathcal{B}_{0}} \tilde{\boldsymbol{B}}_{A}: \tilde{\boldsymbol{H}}_{A} \mathrm{~d} V+\int_{\mathcal{B}_{0}} p_{A}\left(J(\tilde{\boldsymbol{C}})-\tilde{J}_{A}\right) \mathrm{d} V+\int_{\mathcal{B}_{0}} \tilde{p}_{A} \tilde{J}_{A} \mathrm{~d} V
$$

with $\Psi_{\mathrm{M}}\left(\tilde{\boldsymbol{C}}, \tilde{\boldsymbol{H}}, \tilde{J}, \Theta, \boldsymbol{C}_{v}\right)$ and $\Psi_{\mathrm{F}_{i}}\left(\tilde{\boldsymbol{C}}_{A}, \tilde{\boldsymbol{H}}_{A}, \tilde{J}_{A}, \Theta, \boldsymbol{M}_{i}\right)$. Considering the different dependencies of the different parts of the strain energy function (see Table 1), the superimposed fields can now be designed (see Reference [8]). The superimposed fields of the CoCoA element read

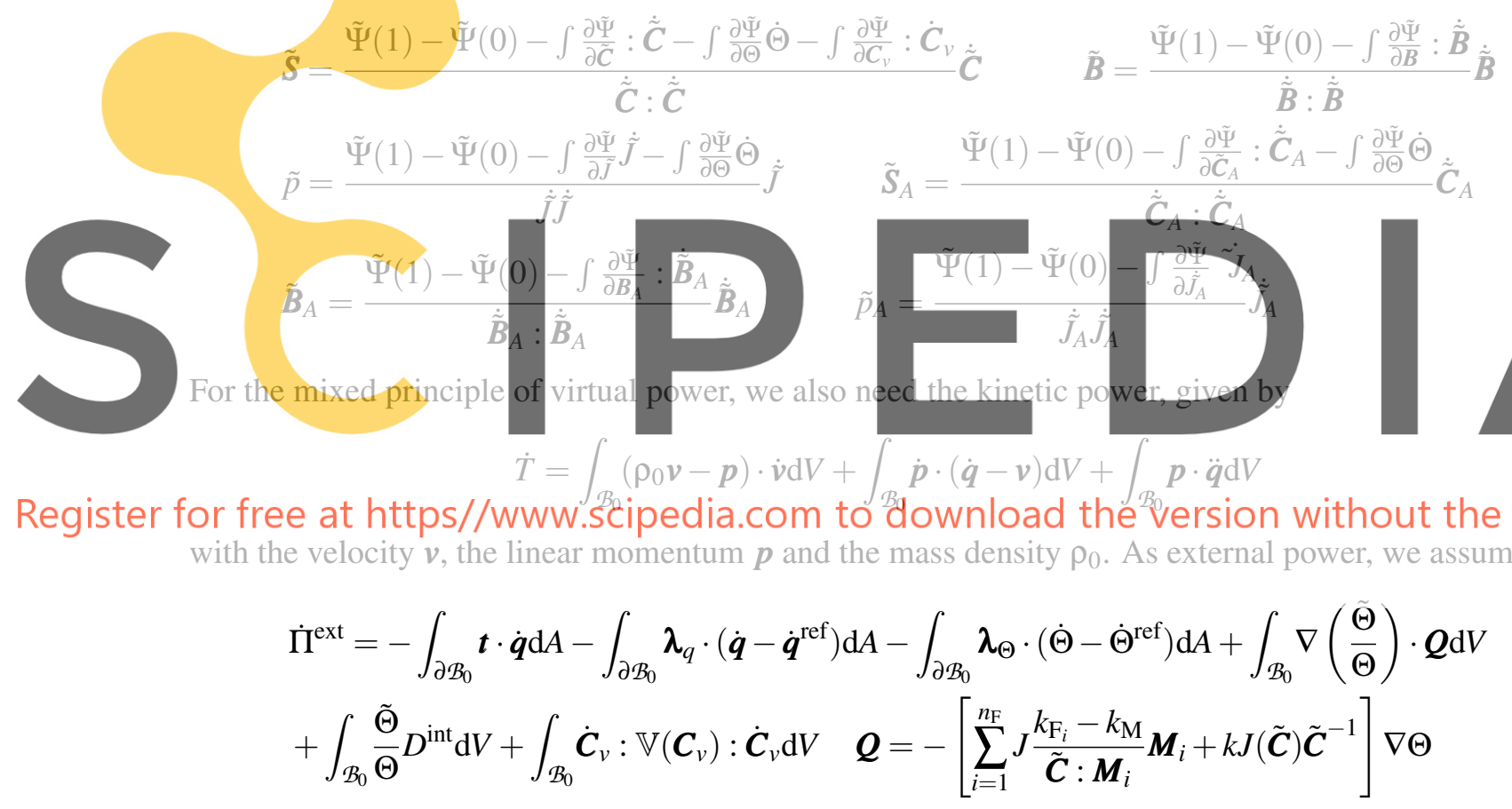

Here, $\boldsymbol{Q}$ denotes the Piola heat flux vector derived from Duhamel's law (see Reference [8]), where $k_{\mathrm{M}}$ and $k_{\mathrm{F}_{i}}$ denotes the material conductivity coefficients for matrix and fibers. For the SKA and CoCoA element, the dependence of the fiber part on $\boldsymbol{Q}$ changes from $\tilde{\boldsymbol{C}}$ to $\tilde{\boldsymbol{C}}_{A}$. Here, $\dot{\boldsymbol{q}}^{\text {ref }}$ denotes the time evolution of a prescribed boundary displacement with the Lagrange multiplier $\boldsymbol{\lambda}_{q}$, and $\dot{\Theta}^{\text {ref }}$ the time evolution of a prescribed boundary temperature with the Lagrange multiplier $\boldsymbol{\lambda}_{\Theta}$. The vector $\boldsymbol{t}$ denotes the traction load on the Neumann boundary. The non-negative internal viscous dissipation $D^{\text {int }}$ is given by

$$
D^{\mathrm{int}}=\dot{\boldsymbol{C}}_{v}: \mathbb{V}\left(\boldsymbol{C}_{v}\right): \dot{\boldsymbol{C}}_{v} \quad \mathbb{V}\left(\boldsymbol{C}_{v}\right)=\frac{1}{4}\left(V_{\mathrm{vol}}-\frac{V_{\mathrm{dev}}}{n_{\mathrm{dim}}}\right) \boldsymbol{C}_{v}^{-1} \otimes \boldsymbol{C}_{v}^{-1}+\frac{V_{\mathrm{dev}}}{4} \mathbb{I}_{\mathrm{s}}: \boldsymbol{C}_{v}^{-1} \otimes \boldsymbol{C}_{v}^{-1},
$$


with the fourth-order symmetric projection tensor $\mathbb{I}_{\mathrm{s}}$ and the viscosity constants $V_{\mathrm{dev}}$ and $V_{\mathrm{vol}}$, respectively. The total energy balance $\mathcal{H}$ thus reads

$$
\begin{aligned}
\dot{\mathcal{H}}=\dot{T} & \dot{\boldsymbol{q}}, \dot{\boldsymbol{v}}, \dot{\boldsymbol{p}})+\dot{\Pi}^{\mathrm{ext}}\left(\dot{\boldsymbol{q}}, \boldsymbol{\lambda}_{q}, \boldsymbol{\lambda}_{\Theta}, \dot{\boldsymbol{C}}_{v}, \tilde{\Theta}, \dot{\Theta}\right) \\
& +\dot{\Pi}^{\mathrm{int}}\left(\dot{\boldsymbol{q}}, \tilde{\Theta}, \dot{\eta}, \dot{\boldsymbol{C}}_{v}, \dot{\tilde{\boldsymbol{C}}}, \dot{\tilde{\boldsymbol{C}}}_{A}, \dot{\boldsymbol{H}}, \dot{\tilde{\boldsymbol{H}}}_{A}, \dot{\tilde{J}}_{,}, \dot{\tilde{J}}_{A}, \boldsymbol{S}, \boldsymbol{S}_{A}, \boldsymbol{B}, \boldsymbol{B}_{A}, p, p_{A}\right)
\end{aligned}
$$

The superimposed fields $\left(\tilde{\boldsymbol{S}}, \tilde{\boldsymbol{B}}, \tilde{p}, \tilde{\boldsymbol{S}}_{A}, \tilde{\boldsymbol{B}}_{A}, \tilde{p}_{A}\right)$, the viscous dissipation $D^{\text {int }}$ as well as the Piola heat flux vector $\boldsymbol{Q}$ are defined as parameters not as arguments. By variation with respect to the variables in the argument of Eqn. (13), that is $\int_{T} \boldsymbol{\delta}_{*} \dot{\mathcal{H}} \mathrm{d} t \equiv \int_{T}\left[\boldsymbol{\delta}_{*} \dot{T}+\delta_{*} \dot{\Pi}^{\text {ext }}+\delta_{*} \dot{\Pi}^{\text {int }}\right] \mathrm{d} t=0$, we obtain the weak forms

$$
\begin{aligned}
& \int_{T} \int_{\mathcal{B}_{0}}[\operatorname{Div}[\boldsymbol{F} \boldsymbol{S}]-\dot{\boldsymbol{p}}] \cdot \delta \dot{\boldsymbol{q}} \mathrm{d} V \mathrm{~d} t=0 \quad \int_{T} \int_{\partial \mathcal{B}_{0}}\left[-\boldsymbol{t}-\boldsymbol{\lambda}_{q}\right] \cdot \delta \dot{\boldsymbol{q}} \mathrm{d} A \mathrm{~d} t=0 \quad \int_{T} \int_{\mathcal{B}_{0}}\left[\frac{1}{\rho_{0}} \boldsymbol{p}-\dot{\boldsymbol{q}}\right] \cdot \delta \dot{\boldsymbol{v}} \mathrm{d} V \mathrm{~d} t=0 \\
& \int_{T} \int_{\mathcal{B}_{0}}\left[\eta+\frac{\partial \Psi}{\partial \Theta}\right] \delta \dot{\Theta} \mathrm{d} V \mathrm{~d} t=0 \quad \int_{T} \int_{\partial \mathcal{B}_{0}}\left[-\boldsymbol{\lambda}_{\Theta}\right] \cdot \delta \dot{\Theta} \mathrm{d} A \mathrm{~d} t=0 \quad \int_{T} \int_{\mathcal{B}_{0}}\left[\frac{\operatorname{Div}[\boldsymbol{Q}]}{\Theta}+\frac{D^{\mathrm{int}}}{\Theta}+\dot{\eta}\right] \delta \tilde{\Theta} \mathrm{d} V \mathrm{~d} t=0 \\
& \int_{T} \int_{\partial \mathcal{B}_{0}}\left[\dot{\tilde{q}}-\dot{q}^{\mathrm{ref}}(t)\right] \cdot \delta \lambda_{q} \mathrm{~d} A \mathrm{~d} t=0 \quad \int_{T} \int_{\partial \mathcal{B}_{0}}\left[\dot{\Theta}-\dot{\Theta}^{\mathrm{ref}}(t)\right] \cdot \delta \lambda_{\Theta} \mathrm{d} A \mathrm{~d} t=0 \\
& \int_{T} \int_{\mathcal{B}_{0}} \frac{1}{2}[\dot{\tilde{\boldsymbol{C}}}-\dot{\boldsymbol{C}}]: \delta S \mathrm{~d} V \mathrm{~d} t=0 \quad \int_{T} \int_{\mathcal{B}_{0}}[\Theta-\tilde{\Theta}] \delta \dot{\eta} \mathrm{d} V \mathrm{~d} t=0 \\
& \int_{T} \int_{\mathcal{B}_{0}}\left[\frac{1}{2} \boldsymbol{S}-\left(\frac{\partial \Psi}{\partial \tilde{\boldsymbol{C}}}+\tilde{\boldsymbol{S}}+\overline{\boldsymbol{S}}\right)\right]: \delta \dot{\tilde{\boldsymbol{C}}} \mathrm{d} V \mathrm{~d} t=0 \quad \int_{T} \int_{\mathcal{B}_{0}}\left[\frac{\partial \Psi}{\partial \boldsymbol{C}_{v}}+\dot{\boldsymbol{C}}_{v}: \mathbb{V}\left(\boldsymbol{C}_{v}\right)\right]: \delta \dot{\boldsymbol{C}}_{v} \mathrm{~d} V \mathrm{~d} t=0
\end{aligned}
$$

For the CoCoA element the remaining weak forms are
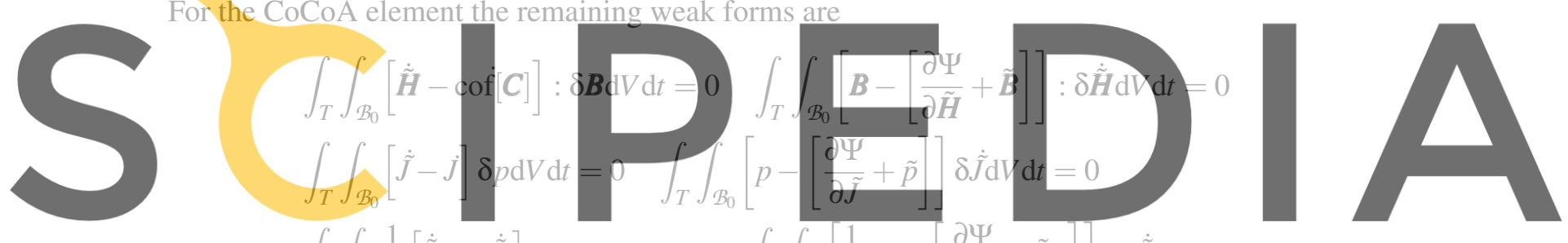

Register for free at ${ }^{T}$ tetps//www.scipedia.com to download the version without the watermark

$$
\begin{aligned}
& \int_{T} \int_{\mathcal{B}_{0}}\left[\dot{H}_{A}-\operatorname{cof}[C]\right]: \delta B_{A} \mathrm{~d} V \mathrm{~d} t=0 \quad \int_{T} \int_{\mathcal{B}_{0}}\left[B_{A}-\left[\frac{\partial \Psi}{\partial \tilde{H}_{A}}+\tilde{B}_{A}\right]\right]: \delta \dot{H}_{A} \mathrm{~d} V \mathrm{~d} t=0 \\
& \int_{T} \int_{\mathcal{B}_{0}}\left[\dot{\tilde{J_{A}}}-\dot{J}\right] \delta p_{A} \mathrm{~d} V \mathrm{~d} t=0=0 \quad \int_{T} \int_{\mathcal{B}_{0}}\left[p_{A}-\left[\frac{\partial \Psi}{\partial \tilde{J}_{A}}+\tilde{p}_{A}\right]\right] \delta \dot{\tilde{J}}_{A} \mathrm{~d} V \mathrm{~d} t=0
\end{aligned}
$$

with the additional stress tensor

$$
\overline{\boldsymbol{S}}=\boldsymbol{B}: \mathbb{P}+\frac{p}{2 J(\tilde{\boldsymbol{C}})} \operatorname{cof}[\tilde{\boldsymbol{C}}]+\frac{1}{2} \boldsymbol{S}_{A}+\boldsymbol{B}_{A}: \mathbb{P}+\frac{p_{A}}{2 J(\tilde{\boldsymbol{C}})} \operatorname{cof}[\tilde{\boldsymbol{C}}] \quad \mathbb{P}=\frac{\partial \operatorname{cof}[\tilde{\boldsymbol{C}}]}{\partial \tilde{\boldsymbol{C}}}
$$

In the last step, we transform the integrals to a reference element and discretize all quantities over the element in space and time. For the shape functions in space $\mathrm{N}$ we use Lagrangian shape functions (see Reference [6]). For the shape functions in time we use Lagrangian shape functions as well (see Reference [8]).

$$
M_{i}(\alpha)=\prod_{\substack{j=1 \\ j \neq i}}^{k+1} \frac{\alpha-\alpha_{j}}{\alpha_{i}-\alpha_{j}}, 1 \leq i \leq k+1 \quad \tilde{M}_{i}(\alpha)=\prod_{\substack{j=1 \\ j \neq i}}^{k} \frac{\alpha-\alpha_{j}}{\alpha_{i}-\alpha_{j}}, 1 \leq i \leq k
$$


The time rate variables and mixed fields $\left(\boldsymbol{q}, \boldsymbol{v}, \boldsymbol{p}, \tilde{\Theta}, \Theta, \eta, \boldsymbol{C}_{v}, \tilde{\boldsymbol{C}}, \tilde{\boldsymbol{C}}_{A}, \tilde{\boldsymbol{H}}, \tilde{\boldsymbol{H}}{ }_{A}, \tilde{J}, \tilde{J}_{A}\right)$ are approximated with

$$
(\bullet)^{e, h}=\sum_{I=1}^{k+1} \sum_{A=1}^{n_{n o}} M_{I}(\boldsymbol{\alpha}) \mathrm{N}^{A}(\boldsymbol{\xi})(\bullet)_{I}^{e A} \quad\left((\dot{\bullet})^{e, h}=\frac{1}{h_{n}} \sum_{I=1}^{k+1} \sum_{A=1}^{n_{n o}} M_{I}^{\prime}(\boldsymbol{\alpha}) \mathrm{N}^{A}(\boldsymbol{\xi})(\bullet)_{I}^{e A}\right)
$$

and Lagrangian multipliers and variation fields $\left(\boldsymbol{\lambda}_{q}, \boldsymbol{\lambda}_{\Theta}, \boldsymbol{S}, \boldsymbol{S}_{A}, \boldsymbol{B}, \boldsymbol{B}_{A}, p, p_{A}, \boldsymbol{\delta}_{*} \bullet\right)$ with

$$
(\bullet)^{e, h}=\sum_{I=1}^{k} \sum_{A=1}^{n_{n o}} \tilde{M}_{I} \mathrm{~N}^{A}(\bullet)_{I}^{e A}
$$

Here, $k$ is the polynomial degree in time and $n_{n o}$ is the number of nodes of the spatial discretization. Each integral that exist are solved with the corresponding Gaussian quadrature rule. The internal variable $\boldsymbol{C}_{v}$ is determined on the element using the Newton-Raphson method, but not at each spatial quadrature point by using spatial finite element shape functions. After eliminating $\boldsymbol{p}$ and $\eta$, we condense out the resulting formulation at the element level to a displacement and temperature formulation (see Reference [2]). Therefore, all mixed fields except $q$ and $\Theta$ are discontinuous at the boundaries of spatial elements. We use our In-House Matlab code fEMcon based on the implementation and ideas shown in Reference [6]. To solve the linear systems of equations we use the Pardiso solver from Reference [9]. For the assembly procedure we use the fast sparse routine shown in Reference [10].
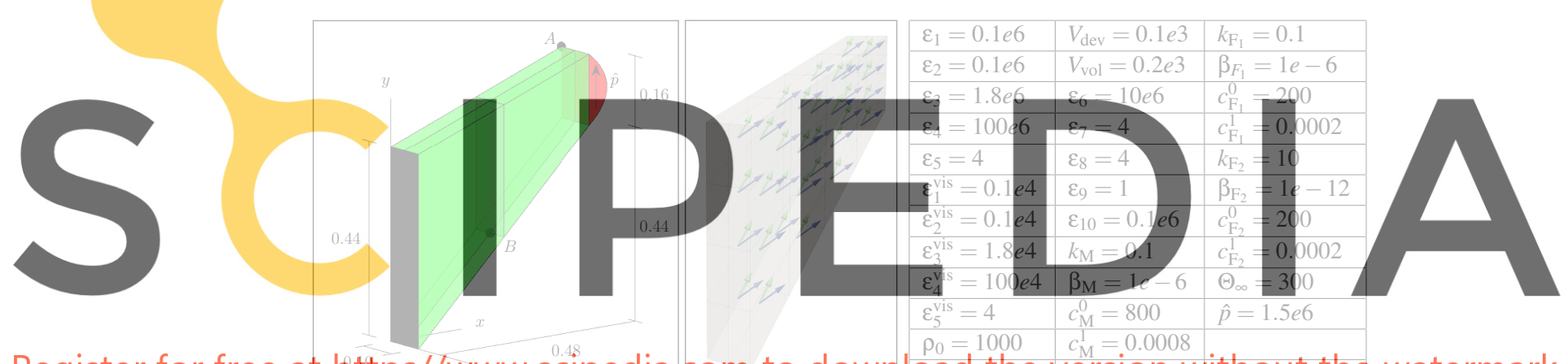

Register for free at https//www.scipedia.com to downlaadotho version witboute the watermark

Figure 1: Geometry, configuration, simulation parameters and fiber directions $\left(\mathbf{a}_{0}^{1}\right)^{T}=\left[\begin{array}{lll}1 & 1 & 1\end{array}\right]$ (blue) and $\left(\mathbf{a}_{0}^{2}\right)^{T}=$ $\left[\begin{array}{lll}1 & 1 & 0\end{array}\right]$ (green) of cooks cantilever beam for $k=1$.

\section{NUMERICAL EXAMPLES}

As first numerical example serves Cook's cantilever beam with a quadratic distribution of an in-plane load on the Neumann boundary. The geometry, configuration and simulation parameters can be found in Figure 1. The corresponding strain energy functions are

$$
\begin{aligned}
& \Psi_{\mathrm{M}}^{\text {iso }}=\frac{\varepsilon_{1}}{2}(\operatorname{tr}[\boldsymbol{C}])^{2}+\frac{\varepsilon_{2}}{2}(\operatorname{tr}[\operatorname{cof}[\boldsymbol{C}]])^{2}-\varepsilon_{3} \ln (J) \quad \Psi_{\mathrm{M}}^{\mathrm{vol}}=\frac{\varepsilon_{4}}{2}\left(J^{\varepsilon_{5}}+J^{-\varepsilon_{5}}-2\right) \\
& \Psi_{\mathrm{F}_{1}}^{\text {ela }}=\varepsilon_{6}\left(\frac{1}{\varepsilon_{7}+1}\left(\operatorname{tr}\left[\boldsymbol{C} \boldsymbol{M}_{1}\right]\right)^{\varepsilon_{7}+1}+\frac{1}{\varepsilon_{8}+1}\left(\operatorname{tr}\left[\operatorname{cof}[\boldsymbol{C}] \boldsymbol{M}_{1}\right]\right)^{\varepsilon_{8}+1}+\frac{1}{\varepsilon_{9}} \operatorname{det}[\boldsymbol{C}]^{-\varepsilon_{9}}\right) \\
& \Psi_{\mathrm{F}_{2}}^{\text {ela }}=\frac{\varepsilon_{10}}{2}\left(\operatorname{tr}\left[\boldsymbol{C} \boldsymbol{M}_{2}\right]-1\right)^{2} \quad \Psi_{X}^{\text {cap }}=c_{X}^{0}\left(1-\Theta_{\infty} c_{X}^{1}\right)\left(\Theta-\Theta_{\infty}-\Theta \ln \frac{\Theta}{\Theta_{\infty}}\right)-\frac{1}{2} c_{X}^{0} c_{X}^{1}\left(\Theta-\Theta_{\infty}\right)^{2}
\end{aligned}
$$



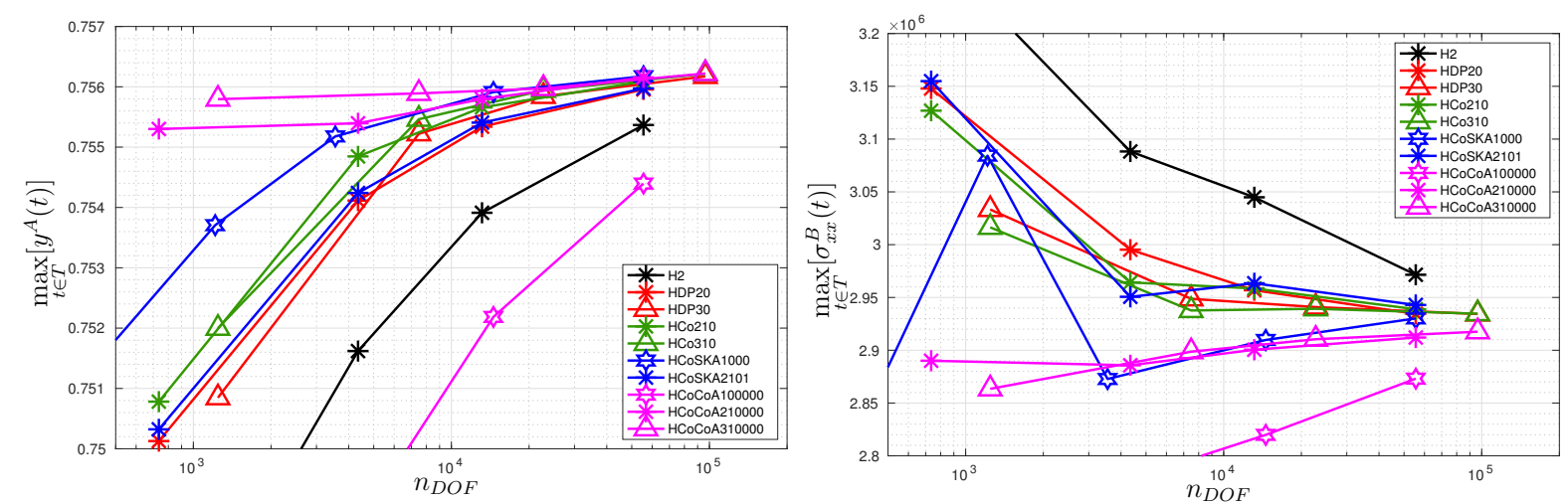

Figure 2: Convergence of the $y$-coordinate on point A and v. Mises equivalent stress $\sigma_{V M}$ on point B for the parameters shown in Figure 1 and $k=1$.

The strain energy function of the matrix $\Psi_{\mathrm{M}}^{\mathrm{iso}}$ and the first fiber $\Psi_{\mathrm{F}_{1}}^{\mathrm{ela}}$ can be found in Reference [2], of the second fiber $\Psi_{\mathrm{F}_{2}}^{\text {ela }}$ in [11] and for the capacitive part the function $\Psi_{X}^{\text {cap }}$ in Reference [8] . Futhermore, the strain energy function of the viscous matrix part is given by $\Psi_{M}^{\mathrm{vis}}=\Psi_{\mathrm{M}}^{\mathrm{iso}}(\boldsymbol{\Lambda})+\Psi_{\mathrm{M}}^{\mathrm{vol}}(\boldsymbol{\Lambda})$. We compare the proposed mixed finite CoCoA-element and the non-standard mixed CoSKA-element and CoFEMelement with the standard displacement element (D) and the displacement pressure element (DP) for hexahedral elements up to cubic order. First we look at the convergence of the y-coordinate at point A and the $\mathrm{v}$. Mises equivalent stress at point B (see Figure 2). The digits in the element name represent the polynomial degrees of the mixed variables in
ments (HCoCoA21000d and HCoCoA310000) with
anisotropic part $\left(\tilde{C}_{A}, \tilde{H}_{A}, \tilde{J}_{A}\right)$ have the highest conv
(HCoSKA1000). This is an interesting case, becau
compared to the other higher order mixed elements
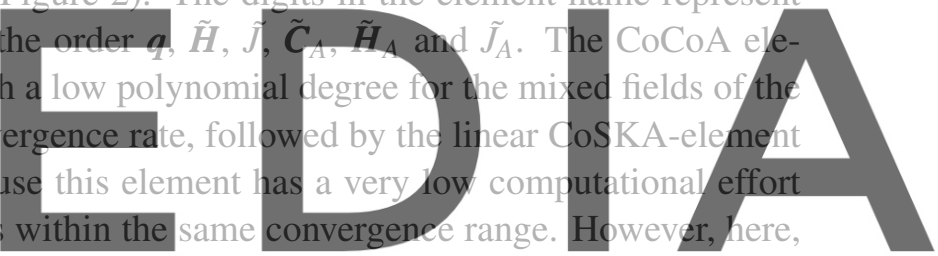

there are certain ozillations or jumps in the stress. After the linear CoSKA-element you will find most

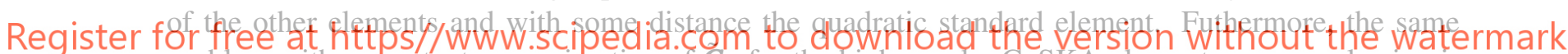
problem with a constant approximation of $C_{A}$ for the higher order CoSKA-elements appears also in visoelastic context (see Reference [5]). In Figure 3 the deformed configuration, the v. Mises equivalent stress $\sigma_{V M}$ and the temperature distribution $\Theta$ are shown. For the fine mesh we get a similar solution, in deformation and stress, for both standard and mixed element. We see that the CoCoA element in the coarse mesh represents the asymmetric bending caused by the fibers, very close to the converged solution. This is also valid for the typical stress distribution of a bending. However, differences between the two meshes can still be seen in the temperature distribution caused by the thermo-mechanical coupling. Next we look on the conservation properties as shown in Figure 4. All shown elements preserve the energy, only the standard element does this slightly worse. The angular momentum is perfectly preserved for all elements, the same is valid for the linear momentum which is not shown. In the last step, we add a second fiber, set the time step size to $h_{n}=0.00025$ and apply a temperature distribution as shown in the first image of Figure 5. For the second fiber, we set the mechanical stiffness to a low value and the conductivity to a high value. As shown in Figure 5, the second fiber ensures that the heat is conducted much faster and in a specific direction. Moreover, we can see in this figure that also here in both cases the energy is conserved and there is no difference between one and two fibers. 


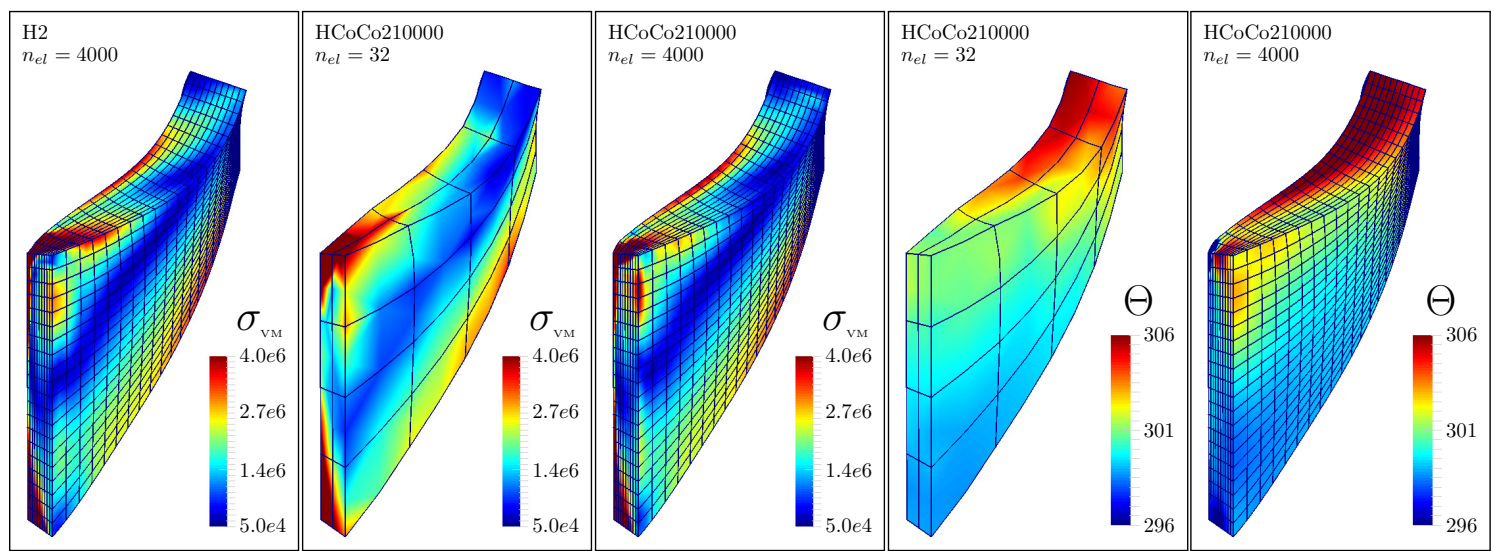

Figure 3: Current configuration, v. Mises equivalent stress $\sigma_{V M}$ and temperature distribution $\Theta$ for the parameters shown in Figure $1, n_{\mathrm{F}}=1$ and $k=1$.
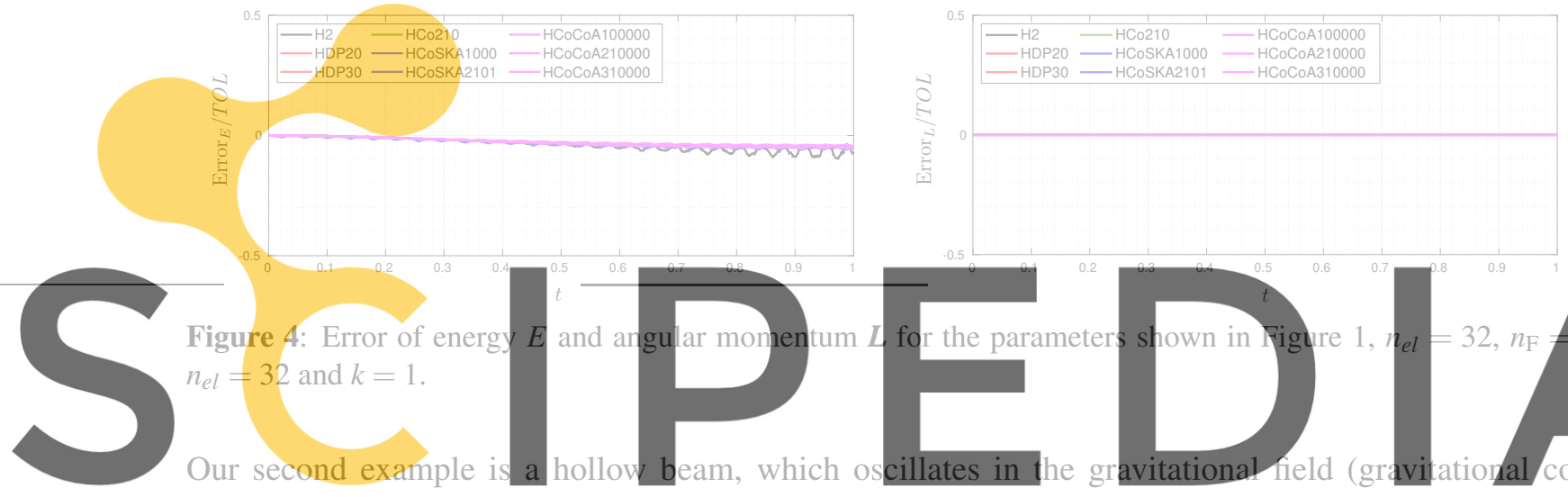

stant $g=9.81$ ) of the earth. The geometry, configuration and simulation parameters can be found in

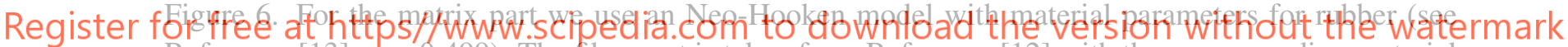

Reference [13], $v=0.499$ ). The fiber part is taken from Reference [12] with the corrosponding material

paramters for a nylon fiber. The strain energy functions of the viscous matrix part and the capacitive part are taken from the first example. All new strain energy parts are given by

$$
\Psi_{\mathrm{M}}^{\text {iso }}=\frac{\varepsilon_{1}}{2}(\operatorname{tr}[\boldsymbol{C}]-3-2 \ln (J)) \quad \Psi_{\mathrm{M}}^{\mathrm{vol}}=\frac{\varepsilon_{2}}{2} \ln (J)^{2} \quad \Psi_{\mathrm{F}_{i}}^{\text {ela }}=\sum_{m=1}^{M_{f}} \frac{\tilde{\mu}_{m}}{\gamma_{m}}\left(\operatorname{tr}\left[\boldsymbol{C M}_{i}\right]^{\frac{\gamma_{m}}{2}}-1\right)-\tilde{\mu}_{m} \ln \left(\operatorname{tr}\left[\boldsymbol{C} \boldsymbol{M}_{i}\right]^{\frac{1}{2}}\right)
$$

The fibers in each wall section are arranged as if they were wrapped around the beam at an angle of 18 degrees. The whole simulation parameters, the geometry and configuration can be found in Figure 6 . Since the material formulation has no cofactor, the corresponding elements are omitted here. First we look at the convergence of the $\mathrm{x}$-coordinate at point $\mathrm{A}$ and the $\mathrm{v}$. Mises equivalent stress at point $\mathrm{B}$ (see Figure 7 left). Here, the best convergence rate is achieved by the HCoSKA2001-element. This is followed by the HDP20-element and then with a significant distance the standard H2-element. If we look at Figure 7 right, we see that all the elements conserves the energy. The difference between the energy errors can be interpreted by the fact that the standard $\mathrm{H} 2$-element is much stiffer and therefore a lower viscous dissipation occurs In the next step, we look at the trajectory of point A for the H2-element and 

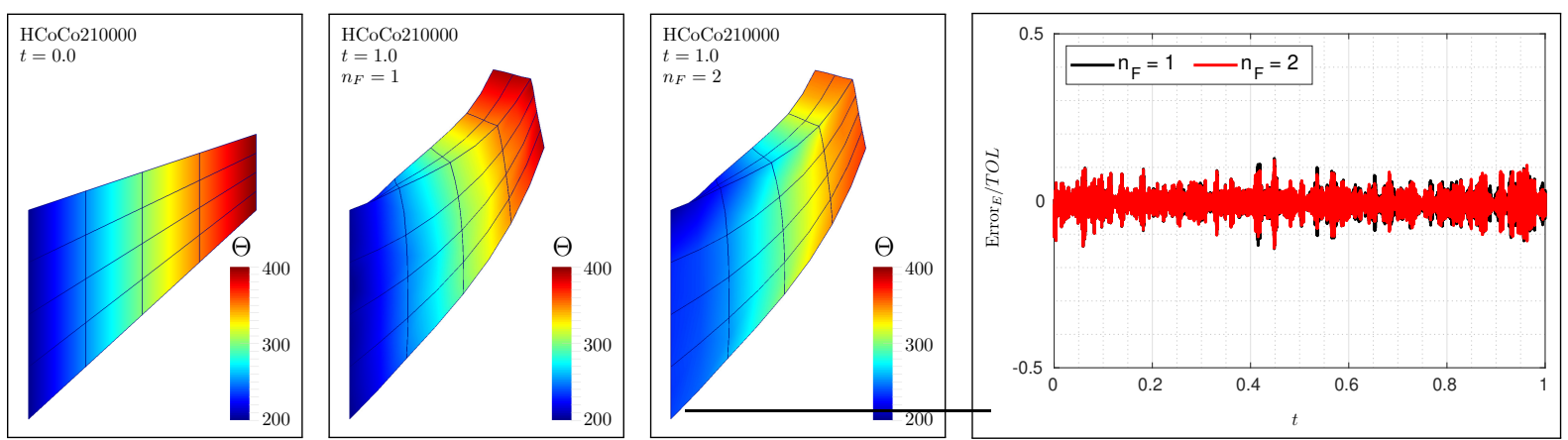

Figure 5: Initial configuration, temperature distribution $\Theta$ and error of energy $E$ for the parameters shown in Figure $1, n_{\mathrm{F}}=2, n_{e l}=32$ and $k=1$.
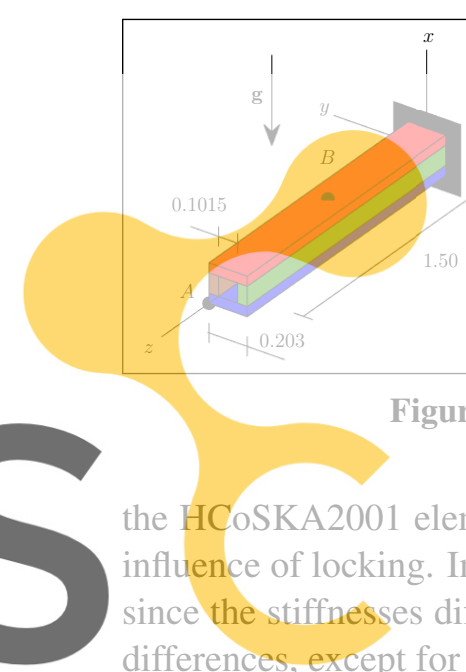

\begin{tabular}{|c|c|c|c|}
\hline$\varepsilon_{1}=0.3360 e 6$ & $c_{\mathrm{M}}^{0}=800$ & $k_{\mathrm{F}_{i}}=0.1$ & $\left(\mathbf{a}_{0}^{1}\right)^{T}=\left[\begin{array}{lll}0 & -0.31 & -0.95\end{array}\right]$ \\
\hline$\varepsilon_{2}=300 e 6$ & $c_{\mathrm{M}}^{1}=0.0008$ & $c_{\mathrm{F}_{1}}^{0}=200$ & $\left(a_{0}^{2}\right)^{T}=\left[\begin{array}{lll}0 & -0.31 & 0.95\end{array}\right]$ \\
\hline$\varepsilon_{1}^{\text {vis }}=0.1 e 6$ & $M_{f}=2$ & $c_{\mathrm{F}_{1}}^{1}=0.0002$ & $\left(\mathbf{a}_{0}^{1}\right)^{T}=\left[\begin{array}{lll}-0.31 & 0 & -0.95\end{array}\right]$ \\
\hline$\varepsilon_{2}^{\mathrm{vis}}=100 e 6$ & $\tilde{\mu}_{1}=40.9118 e 6$ & $\Theta_{\infty}=300$ & $\left(\mathbf{a}_{0}^{2}\right)^{T}=\left[\begin{array}{lll}-0.31 & 0 & 0.95\end{array}\right]$ \\
\hline$V_{\mathrm{dev}}=1 e 5$ & $\tilde{\mu}_{2}=19.0464$ & $\rho_{0}=1100$ & $\left(\mathbf{a}_{0}^{1}\right)^{T}=\left[\begin{array}{lll}0 & 0.31 & -0.95\end{array}\right]$ \\
\hline$V_{\mathrm{dev}}=2 e 5$ & $\gamma_{1}=-31.462 e 6$ & $T=1.0$ & $\left(\mathbf{a}_{0}^{2}\right)^{T}=\left[\begin{array}{lll}0 & 0.31 & 0.95\end{array}\right]$ \\
\hline$k_{\mathrm{M}}=0.1$ & $\gamma_{2}=-165.04$ & $h_{n}=0.0005$ & $\left(\mathbf{a}_{0}^{1}\right)^{T}=\left[\begin{array}{lll}0.31 & 0 & -0.95\end{array}\right]$ \\
\hline$\beta_{\mathrm{M}}=1 e-12$ & $\beta_{\mathrm{F}_{i}}=1 e-12$ & $T O L=1 e-2$ & $\left(\mathbf{a}_{0}^{2}\right)^{T}=\left[\begin{array}{lll}0.31 & 0 & 0.95\end{array}\right]$ \\
\hline
\end{tabular}

Figure 6: Geometry, configuration, simulation parameters of hollow beam.

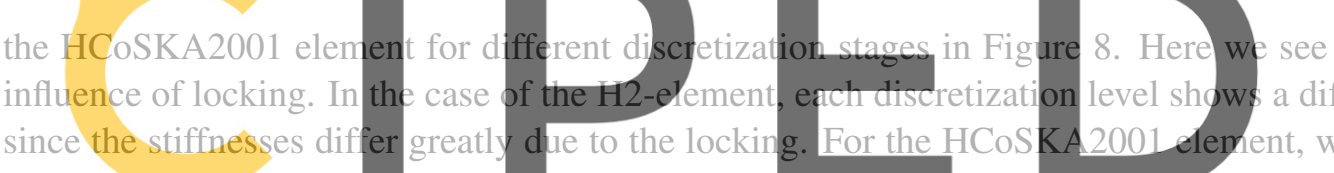
differences, except for the coarsest discretization.
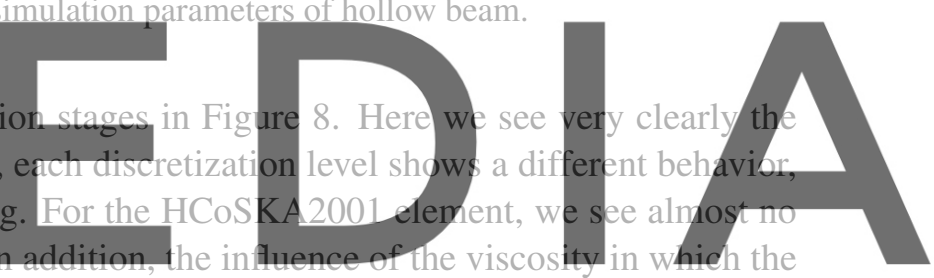

oscillation amplitude decreases can also be seen for this element. This is not possible for the standard

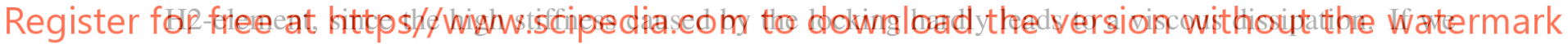
look at the convergence of the maximum stress at point B in Figure 9 (left), we see that the mixed elements converge fast and the standard element does not. If we now look in Table 2 at the results for higher polynomial degrees in time, we see that there is also a certain convergence in time. This is not as large as in space, but the step from $k=1$ to $k=2$ is noticeable. If we now look at the calculation times in table 2, we can see that due to the mixed elements a lower number of degrees of freedom is necessary to obtain a good solution, and thus calculation time can be saved. This is especially relevant in context of higher polynomial degrees in time. In the given case we can save one discretization step and thus save 6.5 times the computation time for $k=2$. For the energy conservation in Figure 9 (right), no differences can be seen between the different polynomial degrees in time.

Our last example is a rotating heatpipe (see Reference [7]) in which we use a carbon fiber reinforced epoxy resin as shown in Reference [11]. Thus, the strain energy parts are given by

$$
\Psi_{\mathrm{M}}^{\mathrm{iso}}=\frac{\varepsilon_{1}}{2}(\operatorname{tr}[\boldsymbol{C}]-3-2 \ln (J)) \quad \Psi_{\mathrm{M}}^{\mathrm{vol}}=\frac{\varepsilon_{2}}{2} \ln (J)^{2} \quad \Psi_{\mathrm{F}_{i}}^{\mathrm{ela}}=\frac{\varepsilon_{3}}{2}\left(\operatorname{tr}\left[\boldsymbol{C} \boldsymbol{M}_{i}\right]-1\right)^{2}
$$

The geometry, configuration and simulation parameters can be found in Figure 10. The heatpipe rotation is implemented with a transient mechanical Dirichlet boundary on both sides $\left(\Omega=1 f_{t}\right.$ with $\left.f_{t}=\frac{1}{T} t\right)$ 

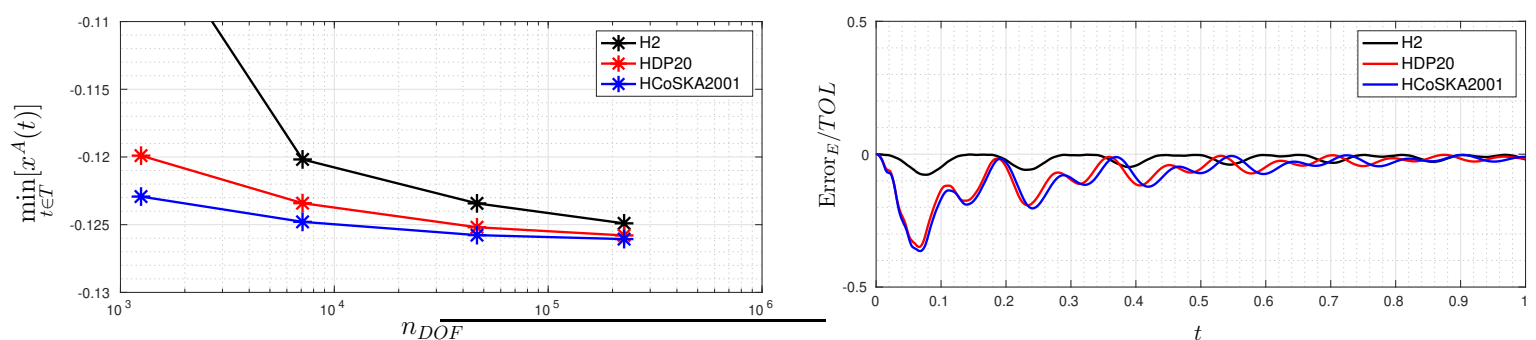

Figure 7: Convergence of the $x$-coordinate on point A and energy error $E\left(n_{e l}=288\right)$ for the parameters shown in Figure 6 and $k=1$.
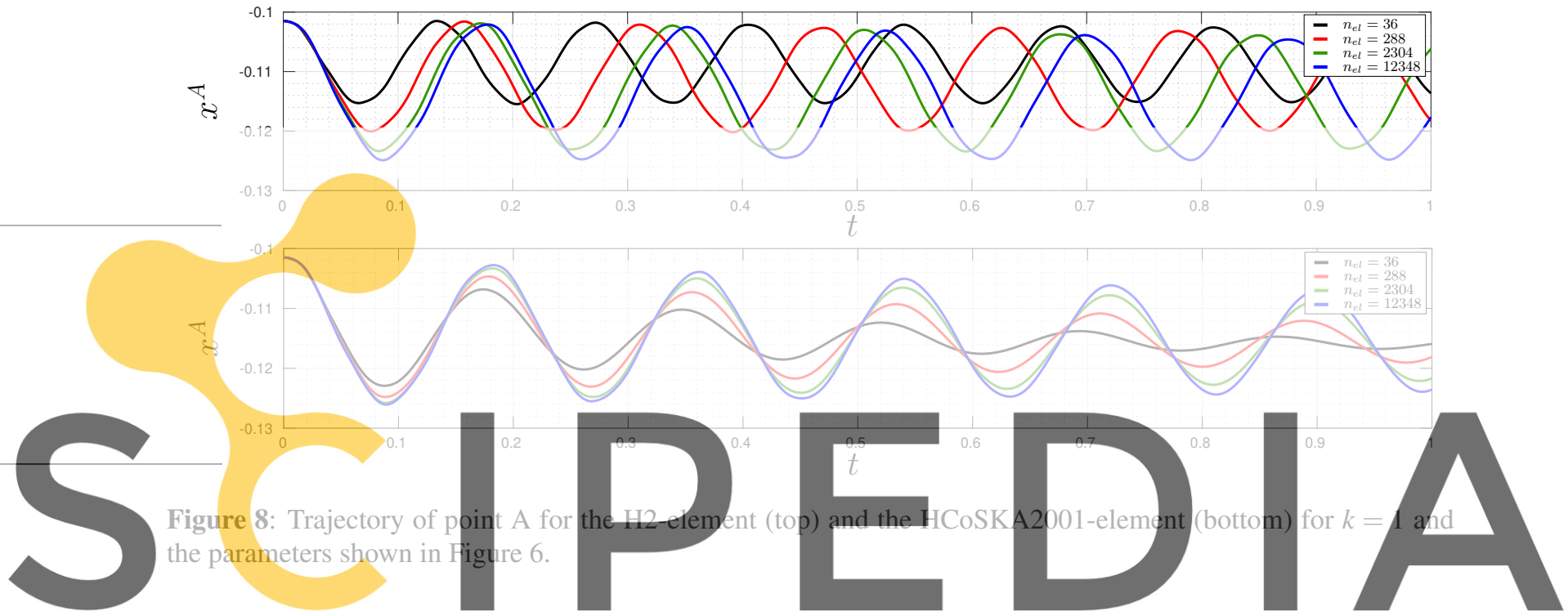

and the time step size is set to $h_{n}=1 e-4$. Additionally, as shown in Figure 10 an inner pressure $(\hat{p})$

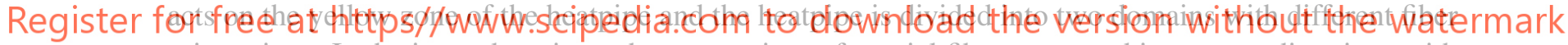
orientations. In the inner domain we have two circumferential fibers arranged in counter directions with an angle of 45 degrees and in the outer domain we have one circumferential fiber. First, we look in

Figure 11 (left) at the convergence of the stress inside the heatpipe. Since the material formulation has no cofactor, the corresponding elements are omitted here also. We can see that the best result shows the CoSKA2011 element, followed by the quadratic displacement-pressure element and the quadratic standard element. The remaining elements converge either very slowly or against another value. We can also see, that here elements with a linear approximation for $\tilde{J}$ generally produce better results. This may be, because the material used is not nearly incompressible. Next we look in Figure 12 at the stress and temperature distribution for the CoSKA2011-element. As expected, the stress is increased due to the inner pressure, especially where the wall thickness is less than under the ribs of the right side. Also on both ribs, we see the temperature is increased in consequence of the viscous dissipation. In the last step, we increase the rotational speed $\left(\Omega=10 f_{t}\right)$, set the time polynomial degree to $k=2$, set the time step size to $h_{n}=1 e-5$ and apply a thermal Dirichlet boundary condition on the ribs on the left side. In Figure 13 we can see, the thermal boundary condition increases the temperature of the ribs on the left side. In addition, the energy is also conserved in this system (see Figure 11 (rigth)) The jumps can be 

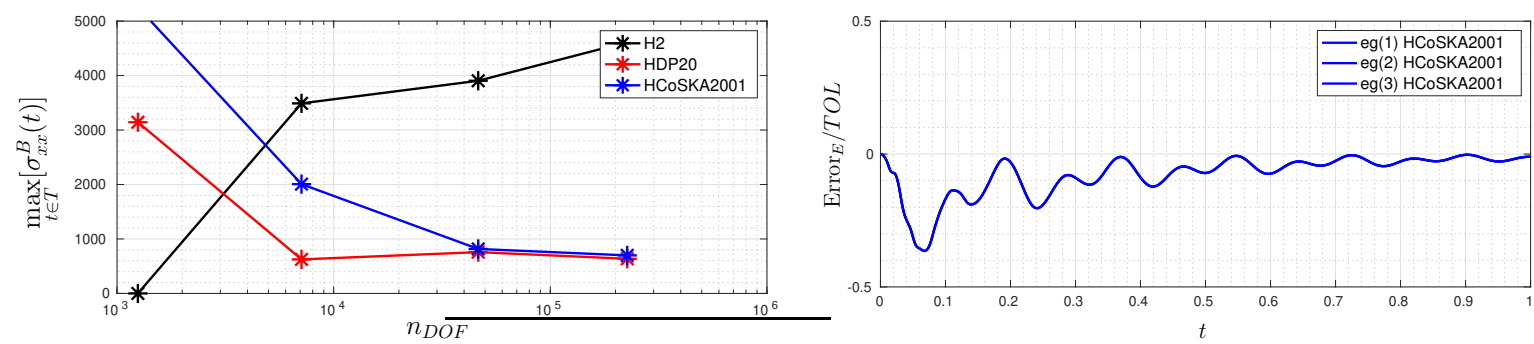

Figure 9: Convergence of $\sigma_{x x}$ on point B and energy error for $k=1,2,3$ for the parameters shown in Figure 6.

Table 2: $\max _{t \in T}\left[\sigma_{x x}^{B}(t)\right]$ for $k=1,2,3$ and the parameters shown in Figure 6 .

\begin{tabular}{|c|c|c|c|}
\hline$n_{e l}$ & $\max _{t \in T}\left[\sigma_{x x}^{B}(t)\right](k=1)$ & $\max _{t \in T}\left[\sigma_{x x}^{B}(t)\right](k=2)$ & $\max _{t \in T}\left[\sigma_{x x}^{B}(t)\right](k=3)$ \\
\hline 36 & $5.2570 e 3$ & $5.1845 e 3$ & $5.2002 e 3$ \\
\hline 288 & $2.0064 e 3$ & $1.9711 e 3$ & $1.9912 e 3$ \\
\hline 2304 & $8.1669 e 2$ & $8.6423 e 2$ & $8.5406 e 2$ \\
\hline 12348 & $6.9841 e 2$ & $6.4615 e 2$ & $6.6118 e 2$ \\
\hline
\end{tabular}

explained by the fact that at this point the newton method changes from one to two steps, because of the linear increasing speed of rotation.

\section{CONCLUSIONS}

We can show the excellent context and we have a hu

internal viscous variabl

coupling and viscous diss momentum time integrate
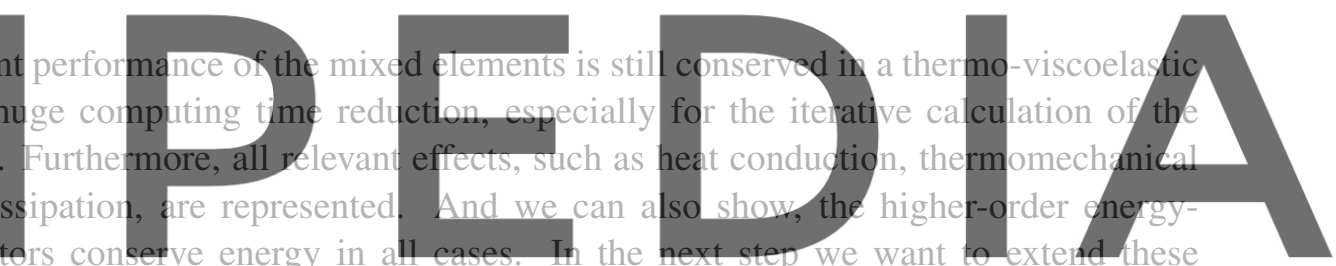

formulations to higher-order gradients to capture the fiber-bending stiffness.

Register for free at https//www.scipedia.com to download the version without the watermark Acknowledgments

The authors thank the 'Deutsche Forschungsgeselischaft (DFG)' for the financial support of this work under the grant GR3297 as well as Matthias Bartelt (GR 3297/2-2) for providing the program.

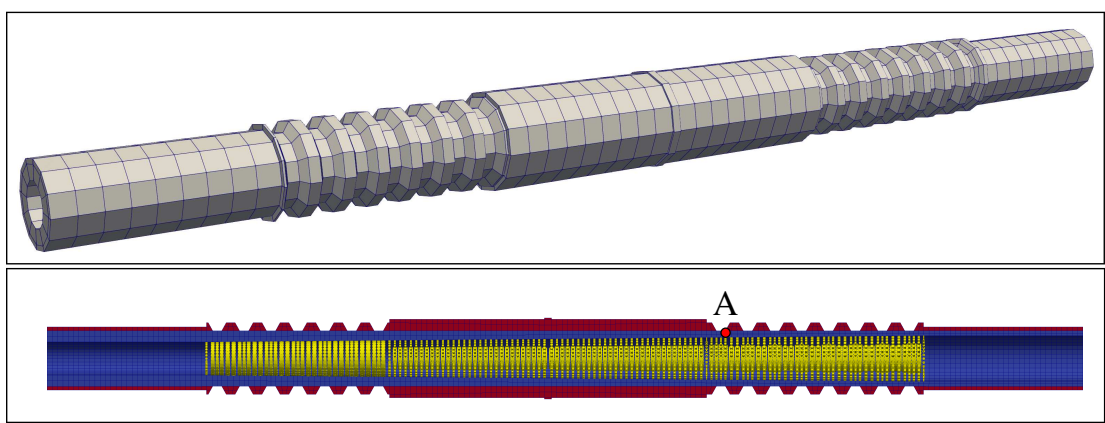

\begin{tabular}{|l|l|}
\hline$\varepsilon_{1}=4.04 e 9$ & $\varepsilon_{3}=800$ \\
\hline$\varepsilon_{2}=5.2 e 9$ & $\beta_{\mathrm{F}_{i}}=1 e-12$ \\
\hline$\varepsilon_{1}^{\mathrm{vis}}=4.04 e 8$ & $k_{\mathrm{F}_{i}}=0.1$ \\
\hline$\varepsilon_{2}^{\mathrm{vis}}=5.2 e 8$ & $c_{\mathrm{F}_{i}}^{0}=200$ \\
\hline$V_{\mathrm{dev}}=1 e 8$ & $c_{\mathrm{F}_{i}}^{1}=0.0002$ \\
\hline$V_{\mathrm{vol}}=2 e 8$ & $\Theta_{\infty}=300$ \\
\hline$k_{\mathrm{M}}=0.5$ & $\rho_{0}=1100$ \\
\hline$\beta_{\mathrm{M}}=5.2 e-14$ & $T=1$ \\
\hline$c_{\mathrm{M}}^{0}=800$ & $T O L=1 e-2$ \\
\hline$c_{\mathrm{M}}^{1}=0.0008$ & $\hat{p}=0.5 e 8 f_{t}$ \\
\hline
\end{tabular}

Figure 10: Geometry, configuration, simulation parameters of rotating heatpipe. 
Table 3: $t_{C P U}$ for $k=1,2,3$ and the parameters shown in Figure 6 on an Intel Core i9-10940X.

\begin{tabular}{|c|c|c|c|}
\hline$n_{D O F}(k=1,2,3)$ & $t_{C P U}(k=1)$ & $t_{C P U}(k=2)$ & $t_{C P U}(k=3)$ \\
\hline $1428,2856,4284$ & 487 & 687 & 1201 \\
\hline $7680,15360,23040$ & 837 & 2741 & 5533 \\
\hline $48480,96960,145440$ & 7860 & 13985 & 38970 \\
\hline $232260,464520,696780$ & 20168 & 90227 & 261256 \\
\hline
\end{tabular}
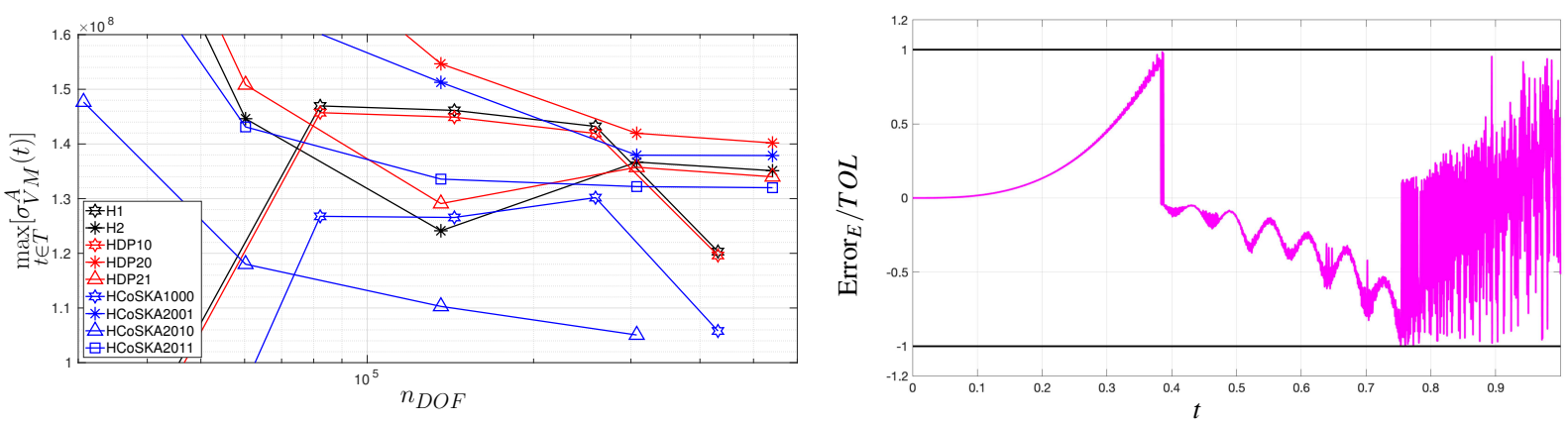

Figure 11: Convergence of v. Mises equivalent stress $\sigma_{V M}$ on point $\mathrm{B}$ for $k=1$ and energy error $E$ for the 2 nd case and $k=2$ and the parameters shown in Figure 10 .

\section{REFERENCES}

[1] Simo, J. C., Taylor, R. L., and Pister, K. S. (1985). Variational and projection methods for the volume constraint in finite deformation elasto-plasticity. Comput. Methods Appl. Mech. Engrg., 51(1-3), 177-208. https://doi.org/10.1016/0045-7825(85)90033-7

[2] Schröder, J., Wriggers, P., and Balzani, D. (2011). A new mixed finite element based on different approximations of the minors of deformation tensors. Comput. Methods Appl. Mech. Engrg., 49:3583-3600.

[3] Schröder, J., Viebahn, V., Wriggers, and P., Balzani, D. (2016). A novel mixed finite element for finite anisotropic elasticity; the SKA-element Simplified Kinematics for Anisotropy. Comput. Methods Appl. Mech. Engrg., 310:475-494.

[4] Bonet, J., Gil, A., Ortigosa, R. (2015). A computational framework for polyconvex large strain elasticity. Computer Methods in Applied Mechanics and Engineering. Volume 283. Pages 10611094. Mathematics and Computers in Simulation, in press, DOI 10.1016/j.matcom.2018.03.0.

[5] Dietzsch, J., and Groß, M. (2018). MIXED FINITE ELEMENT FORMULATIONS FOR THE GALERKIN-BASED TIME INTEGRATION OF FINITE ANISOTROPIC ELASTODYNAMICS, ECCOMAS conference ECCM-ECFD 2018 - 6th European Conference on Computational Mechanics (ECCM 6), Glasgow UK, 11-15 June 2018.

[6] Bartelt, M., Dietzsch, J., and Groß, M. (2018). Efficient implementation of energy conservation for higher order finite elements with variational integrators. Math. Comput. Simulat., 150, 83-121. https://doi.org/10.1016/j.matcom.2018.03.002

[7] Groß, M., and Dietzsch, J. (2017). Variational-based energy-momentum schemes of higher-order 


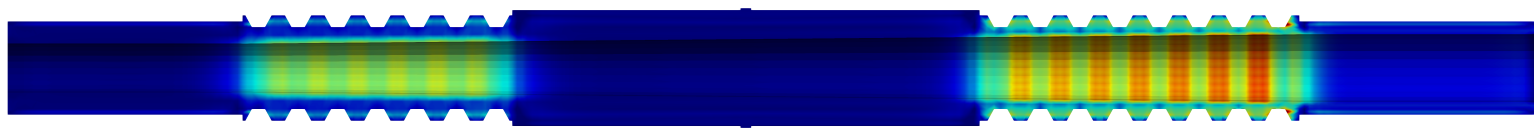

\section{$\sigma_{V M}$}

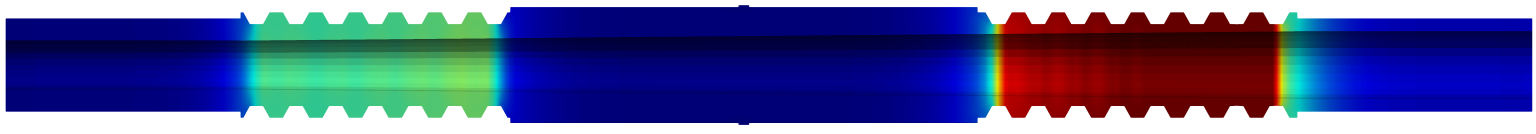

$\Theta$

300 303

Figure 12: V. Mises equivalent stress $\sigma_{V M}$ and temperature distribution $\Theta$ for the CoSKA2011-element, $t=1$, $n_{e l}=8184$, the parameters shown in Figure 10 and $k=1$.

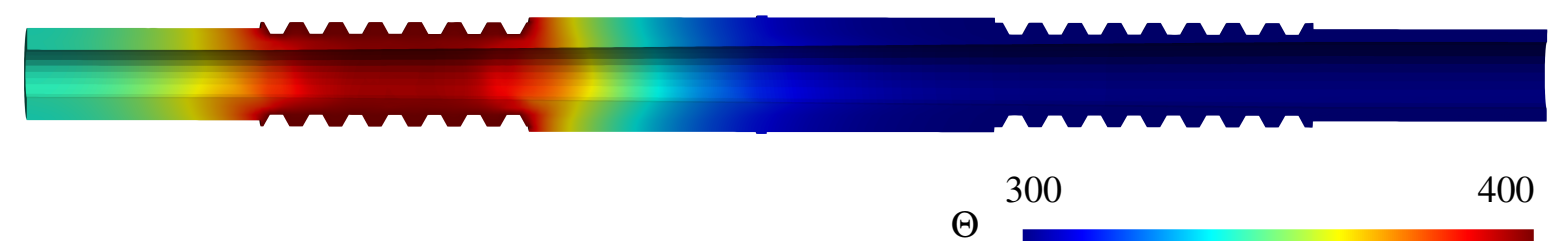

Figure 13: Temperature distribution $\Theta$ for the CoSKA2011-element, $t=1, n_{e l}=8184$, the parameters shown in Figure 10 and $k=2$.

for elastic fiber-reinforced continua. Computer Methods in Applied Mechanics and Engineering, 320, 509-542. https://doi.org/10.1016/j.cma.2017.03.018

[8] Groß, M., Dietzsch, J., and Bartelt, M. (2018). Variational-based higher-order accurate energy-momentum schemes for thermo-viscoelastic fiber-reinforced continua. Comput. Methods Appl. Mech. Engrg., 336, 353-418. https://doi.org/10.1016/j.cma.2018.03.019

[9] Alappat, C., Basermann, A., Bishop, A. R., Fehske, H., Hager, G., Schenk, O., Thies, J., and Wellein, G. (2020). A Recursive Algebraic Coloring Technique for Hardware-efficient Symmetric Sparse Matrix-vector Multiplication. ACM Transactions on Parallel Computing, 7(3). https://doi.org/10.1145/3399732

[10] Engblom, S., and Lukarski, D. (2016). Fast Matlab compatible sparse assembly on multicore computers. Parallel Computing, 56, 1-17. https://doi.org/10.1016/j.parco.2016.04.001

[11] Dal, H., Gültekin, O., Aksu Denli, F., and Holzapfel, G. A. (2017). Phase-Field Models for the Failure of Anisotropic Continua. PAMM, 17(1). https://doi.org/10.1002/pamm.201710027

[12] Markert, B., Ehlers, W., and Karajan, N. (2005). A general polyconvex strain-energy function for fiber-reinforced materials. PAMM, 5(1), 245-246. https://doi.org/10.1002/pamm.200510099

[13] Steinmann, P., Hossain, M., and Possart, G. (2012). Hyperelastic models for rubber-like materials: consistent tangent operators and suitability for Treloar's data. Arch. Appl. Mech., 82(9), 1183-1217. https://doi.org/10.1007/s00419-012-0610-z 